\title{
Chemical Reactions Involving Solids*
}

\section{Physical Basis}

$\mathrm{I}$ TRODUCTORY papers by Profs. N. F. Mott (Bristol) and J. C. Slater (Cambridge, Mass.) dealt with the motion of electrons in polar salts. Electrons can be set free by the absorption of light or by the heat motion of the atoms of a erystal ; however, the absorption of light will not necessarily free an electron; an atom of the crystal may be raised merely into an excited state, without the electron being free to move far away from its original position. An excited atom in a crystal has been termed an 'exciton'; an exciton can move a certain distance through the erystal by transferring its energy from atom to atom, before the energy is all dissipated. The various types of lattice imperfection at which an electron can be trapped were also discussed.

Papers by Prof. J. D. Bernal (London), Prof. G. Hevesy (Copenhagen), H. A. C. McKay (London), Prof. C. Wagner (Darmstadt), Prof. W. Jost (Hanover), Dr. T. P. Hoar and Dr. L. E. Price (Cambridge), and Dr. J. A. A. Ketelaar (Leyden), dealt mainly with the transport of atoms and ions through solids. Experiments on self-diffusion in metals were described, using natural and artificial radioactive materials as indicators. A hypothesis was advanced to account for the small values of the coefficients of self-diffusion, compared with the coefficients of diffusion of one metal into another. Ionic conductivity and diffusion in solid polar salts were discussed in the light of the theory that there exist in a polar salt in thermodynamical equilibrium a certain number of vacant lattice points and a number of ions in interstitial positions. Experimental and theoretical methods of estimating the activation energies necessary for the formation of these configurations were described. Finally an account was given of the behaviour of salts of very high conductivity, for example, $\alpha \cdot \mathrm{Ag}_{2} \mathrm{HgI}_{4}$.

\section{Photochemical Processes}

A number of papers dealt with photochemical reaction in solids. Dr. R. Hilsch and Prof. R. W. Pohl (Göttingen) demonstrated a beautiful experiment in which a transparent crystal of potassium bromide containing $\mathrm{KH}$ in solid solution is coloured blue on exposure to ultra-violet light, the well-known Farbzentren being formed. The efficiency of the photochemical reaction falls rapidly, however, as the temperature is lowered. Prof. J. Eggert and M. Biltz (Leipzig) described experiments on the sensitivity of the silver bromide photographic layers; they considered that Störungstellen in the halide grains have two functions: to serve as nuclei for the formation of the latent image, and, for wave-lengths greater than $500 \mathrm{~m} \mu$, to make possible the absorption of light.

Papers by Dr. C. F. Goodeve and J. A. Kitchener (London) dealt with photosensitization by solids, and by Prof. W. Reinders and Dr. M. C. F. Benkers

* Report of a General Discussion, arranged by the Faraday Society, held in the University of Bristol on April 11-13.
(Delft) with the physical development of the latent image. Dr. Lüppo-Cramer (Jena) contributed a paper on photographic sensitivity, and Prof. F. Weigert (Glasgow) on a new time phenomenon in photographic emulsions.

\section{Thermal Decomposition and the Detonation of Solids}

Prof. S. Roginski (Leningrad), in a classification of the reactions involving solids, showed that sometimes the chemical and sometimes the crystallization steps determine the course of the reaction. Near equilibrium and when $Q+E$ is small or negative, the processes are governed by crystallographic factors, the rate of reaction in such cases being proportional to the surface area of the grains. The decomposition of hydrates and mercuric oxide ( $\mathrm{HgO})$ are good examples of this type. On the other hand, when $Q+E$ is large and the processes are irreversible, the chemical reaction may take control and the reaction penetrate the lattice in an irregular fashion, giving rise to chain reactions. F. R. Meldrum (Bristol) gave an account of the decomposition of nitrogen iodide, which may be of this latter type.

The conditions under which nuclei form and grow on the surface of crystalline solids were dealt with by Prof. W. E. Garner (Bristol), and a distinction was drawn between endothermic and exothermic changes. There is lattice penetration in certain explosives, for example, mercury fulminate, and it is found generally that in the exothermic class, $d p / d t=k p^{n}$, where $n=0 \cdot 8-1$, is obeyed instead of the $2 / 3$ power law. This indicates that these reactions are chain reactions, it being maintained by some investigators that the chains are structural in character and by others that they are analogous to the chain reactions occurring in the combustion of hydrocarbons (Semenoff).

A number of phenomena arise when the rates of formation of the new solid phases are slow. Prof. J. Zawadski and Dr. S. Bretsznajder (Warsaw) showed that spurious equilibria (sorption equilibria) are found in the case of zinc and cadmium carbonates which are due to the slow rate of crystallization of the oxides and carbonates. In the case of hydrates, two factors are involved: (1) the retardation of the rate of reaction by water vapour is greater when the new solid phase is amorphous than when it is crystalline; and (2) water vapour catalyses the crystallization of the new phase (Volmer). Dr. J. Colvin and J. Hume (Leeds) discussed the application of these principles to the appearance of metastable hydrates during dehydration, and showed that the tri-hydrate of copper sulphate is produced when conditions are favourable to the crystallization of this phase. They also gave an account of the TopleySmith effect with $\mathrm{Mn}(\mathrm{COO}){ }_{2} 2 \mathrm{H}_{2} \mathrm{O}$, where a minimum in the rate of dehydration occurs at a water vapour pressure of $0.1 \mathrm{~mm}$. In the course of the discussion, it was agreed that the explanation of this effect given by Volmer, which was based on the above principles, is substantially correct. 
The thermal decomposition of solids of the explosive class passes into a linear propagation of the reaction or deflagration under certain conditions, and the cause of this transition was ascribed to the simultaneous decomposition of a small number of adjacent molecules (Prof. W. E. Garner and Dr. H. Muraour (Paris)). It was pointed out that the linear propagation can arise under conditions where there is a negligible self-heating of the crystals and therefore that the deflagration must be due to some localized event. Dr. W. Taylor and A. Weale (Stevenston), who have studied the initiation of detonation by mechanical shock, put forward a tribo chemical theory of the origin of the detonation wave, ascribing to the action of friction the activation of a number of adjacent molecules which serves as the centre from which detonation can be initiated.

As Muraour pointed out, although the thermal decomposition can pass into deflagration, it is not possible in all cases for deflagration to pass into detonation, and Taylor and Weale ascribed this to the necessity for a critical stress which must be set up before detonation can occur. This critical stress varies in magnitude from case to case, being very small for explosions of the initiating class but possessing a high value in the case of typical high explosives. This stress cannot arise in all cases from the thermal decomposition but can be brought about by initiation with a more sensitive explosive. The view was expressed in the discussion, in which M. Polanyi, W. Jost, W. Payman and J. D. Bernal took part, that under the high pressures in the detonation wave, 350 tons/in. ${ }^{2}$ and more, the molecular compression would be sufficiently high to cause chemical reaction, the effect of the stress being such as to cause the disappearance of the energy barrier separating the reactants from their products. No general conclusion was reached on this matter. it being considered that other factors such as temperature may play some part in the propagation of the wave. Dr. T. Carlton Sutton (London) brought forward evidence in the case of tetryl that the heat of detonation increases step-wise with increasing density of loading.

\section{Layer lattices in Solid Carbon}

The unique electronic structure of the carbon atom which is responsible for its extensive organic chem. istry also confers upon solid carbon peculiar properties of considerable interest and fundamental importance. The advent of X-ray methods of studying the solid state has made possible a much deeper insight into the chemical nature and reactivity of black carbon, and the papers discussed in the section of the discussion dealing with this aspect suggested that the near future will witness a rapid development of this solid phase chemistry, which originated with Fredenhagen's discovery of the alkali-graphites some ten years ago.

Already the list of inter-layer lattice compounds is of considerable length, and although the nature of some of the compounds is still somewhat obscure, their further study is likely to give results of considerable importance. Prof. U. Hofmann (Rostock) deseribed the formation of salts of graphite and strong acids. The peculiar step-wise manner in which these salts decompose (similar to the manner in which $\mathrm{C}_{8} \mathrm{~K}$ passes into $\mathrm{C}_{16} \mathrm{~K}$ ) suggests that there is a fundamental electronic connexion between the layer planes of the graphite crystal lattice. Prof. O. Ruff (Breslau) put forward certain suggestions concerning the structure of 'amorphous' carbon and discussed the reactions of carbon with oxygen, fluorine, carbon dioxide and with metals. He also exhibited specimens of graphite monofluoride prepared by himself and Bretschneider and demonstrated the decomposition of the substance on warming. The reaction (Verpuffen) was very reminiscent of the decomposition of graphitic oxide. Prof. H. L. Riley (Newcastle-upon-Tyne) directed attention to the abrupt changes which occur in the properties of the products of carbonizing various organic substances at temperatures in the neighbourhood of $700^{\circ} \mathrm{C}$. $\mathrm{He}$ discussed particularly the susceptibility of various carbons to oxidation to chromic-phosphoric acid and the changes which occur in the strength of coke during carbonization. Dr. H. Thiele (Kiel) discussed the oxidation of black carbon in various electrolytes at room temperature, and demonstrated the swelling of a graphite electrode when anodically polarized in concentrated sulphuric acid, and also the peculiar crackling which occurs when a hard carbon electrode is treated in a similar manner. He referred to the various stages in the oxidation: (1) primary oxide, (2) swollen oxide, (3) blue graphitic oxide and graphitic acid and also humic acid.

The problems connected with the kinetics of earbon combustion are likely to become less obscure as our knowledge of the chemical nature of black carbon increases. In spite of extensive experimental work, the exact mechanism of carbon oxidation is still obscure. Prof. V. Sihvonen (Helsingfors) discussed the influence of keto- and ketene groups and of adsorbed molecules and ions and the mechanism of carbon oxidation. Basing his views on extensive experimental work on the reduction of carbon dioxide, adsorption of oxygen, combustion in oxygen, effect of water vapour and the anodic oxidation of carbon both in electrolytes and in the gas phase, he advanced comprehensive theories as to the mechanism of the breakdown of the carbon lattice. Dr. Lothar Meyer (Höllriegelskreuth, near Munich) described some of his ingenious experiments on carbon combustion at low pressures. The technique employed, following the static experiments of Langmuir, consists in streaming the oxidizing gas at a pressure of a few thousandths of a millimetre of mercury, at a high velocity, over a carbon filament heated electrically, so reducing considerably the chances of secondary reactions occurring. The results obtained with oxygen, carbon dioxide, and water vapour were described. The part played by surface oxides in the oxidation of carbon was discussed by Dr. R. F. Strickland-Constable (Oxford). Although the exact nature of the oxide is still un. certain, there appears to be little doubt that some form of solid oxide plays an important intermediate part in carbon combustion. The views of Rhead and Wheeler put forward twenty-five years ago, and the recent views of Eucken on the mechanism of carbon combustion, support this idea. StricklandConstable also described experiments on the oxidation of carbon in nitrous oxide. J. D. Lambert (Oxford) discussed briefly the effect of certain metals on the oxidation process.

The paper by Prof. E. Berl (Pittsburgh) on the formation and properties of activated carbon was taken as read. Only a very limited time remained for discussion. 